\title{
Highlighting tools and technologies for collaborative learning
}

\section{Carolyn P. Rosé ${ }^{1} \cdot$ Nancy Law ${ }^{2} \cdot{\text { Ulrike } \text { Cress }^{3} \cdot \text { Sten Ludvigsen }}^{4}$}

Published online: 23 April 2019

(C) International Society of the Learning Sciences, Inc. 2019

\section{Introduction}

A common thread that runs throughout the four articles of this March 2019 issue is a highlight of tools and technologies. Prior work characterizing a spectrum of technologies relevant for the field have organized the presentation based on different affordances for collaboration (Jeong and Hmelo-Silver 2016). The English Oxford Living dictionary defines a tool as "a device or implement, especially one held in the hand, used to carry out a particular function." The key is the agency taken by the tool user. The four articles of this issue highlight the contrast between technology as a resource in collaborative learning in two distinct roles, where the distinction is in terms of whose agency is at center stage. In the first two articles, the technology highlight is a scaffold for group reflection, in the first case an awareness tool, and in the second case a selfand peer-assessment tool. These articles are in the spirit of many earlier articles in the journal, including recently (Hadwin et al. 2018; Wang et al. 2017a; Näykki et al. 2017), related to scaffolding collaborative processes. In these cases, the relevant affordances are framed in terms of what they enable students to perceive, and how these perceptions aid in regulatory processes within groups. In contrast, in the second two articles the technology highlight provides affordances for student expression and creativity within collaboration. The first of these positions students as designers and investigates the creative process of artifact design where the artifacts are the products that are the target of group activity. In the final article, students again use technology resources creatively, but here the focus is on artifacts created in the midst of collaboration as communicative devices, in other words, multi-modal representations created by students to aid in their intersubjective communicative processes.

Carolyn P. Rosé

cp3a@andrew.cmu.edu

Carnegie Mellon University Language Technologies Institute and HCI Institute, Pittsburgh, PA, USA

2 University of Hong Kong, Hong Kong, China

3 Leibniz-Institut für Wissensmedien (Knowledge Media Research Center), Tübingen, Germany

4 Faculty of Educational Sciences, University of Oslo, Oslo, Norway 


\section{CSCL community connection}

Since the ICLS 2016 conference when the bylaws change to introduce a mechanism through which the ISLS could more proactively maintain the diversity of its board of directors, awareness has been raised to the importance of taking steps to affirm diversity at all levels, including regional representation, disciplinary representation, and gender representation. Synergistic with that effort, the society has also taken steps to highlight and affirm equity in all respects as an important area of research. Prior to the 2016 bylaws vote, a survey of the CSCL community revealed that though the inclusion of technology is one of the defining characteristics of a contribution to the CSCL conference and the ijCSCL, only $10 \%$ of the membership of the CSCL community identified Computer Science as their core discipline. Though worrisome, this statistic makes sense when one considers that published work in CSCL fronts issues related to collaboration and learning from the perspective of Education and Psychology, and relegates tools and technologies to the background.

The articles in this issue each make substantive contributions to our understanding of collaboration and learning and are valuable to the field for those contributions. With this editorial, our hope is that this issue of the journal also serves to affirm the society's and the journal's valuing of the tools and technologies that serve to enable this important work.

\section{Providing different types of group awareness information to guide collaborative learning}

Lenka Schnaubert and Daniel Bodemer provide an exceptionally rigorous study of the effects of a manipulation of group awareness technology on regulatory practices within collaboration. Group awareness tools support collaborative and metacognitive processes through a three-step process of sensing and assessing group processes, transforming this assessment into an understandable representation that highlights important structure, and then presenting it back to learners through some effective communicative mechanism (Buder and Bodemer 2008). An important characteristic of these tools, and one that sets them apart from other tools and technologies in the area of dashboards in support of learning is the fact that the representations used to feed a message back are designed specifically for learners as the target audience. The article provides a review of this important area of research, outlining the important characteristics of the design of such representations in order to ensure their effectiveness.

During the study, 130 dyads worked together face-to-face using a multi-touch tabletop interface. In this configuration, participants have the opportunity to observe the physical behavior of their partners, which already supports a level of group awareness. The cognitive group awareness intervention was provided in the form of a report of individual assumptions about the correct answers to some task-relevant questions. Metacognitive group awareness support was presented in the form of confidence ratings on aspects of the learning tasks.

An important contribution of the paper is the investigation of the interplay between effects of cognitive and metacognitive support through group awareness tools. While a substantial amount of work in each of these areas exists already, this article fills the gap at the frontier between these two areas in investigating how they can be brought together. What makes this challenging is the extent to which cognitive and metacognitive processes are not independent. The article that specifically investigates the interaction between cognitive and metacognitive guidance in their separate and joint effects on collaborative learning processes and learning 
outcomes. The practical goal of the study is to inform design of improved group awareness tools, making it more clear how to adapt the design to different goals for effect on learners that educators and/or tool designers may have.

The complexity of the target phenomena requires a sophisticated approach to the statistical analysis reported in the article, and Schnaubert and Bodemer do an artful job of managing this complexity and delivering a story that is solid and valuable to the field.

\section{Improving the quality of vocational students' collaboration and knowledge acquisition through instruction and joint reflection}

The featured technology in the next article is again a scaffold for joint reflection on collaborative practices during collaboration, with a focus on twenty-first century skills related to ability to work in teams (Hattie and Donoghue 2016). Specifically, Elise Eshuis, Judith ter Vrugte, Anjo Anjewierden, Lars Bollen, Jakob Sikken, and Ton de Jong investigate the impact of instruction on collaborative practices and tool-mediated joint reflection on those practices during collaboration on the level of appropriation of the practices within collaboration. The surprising finding is the limited utility of training by itself. The current study contributes to a line of research supporting the value of joint reflection in fostering development of collaborative skills during collaborative encounters (Phielix et al. 2010, 2011).

The support for joint reflection featured in this article was designed to highlight what are known as the RIDE rules (Respect, Intelligent collaboration, Deciding together, and Encouraging) (Saab et al. 2005; Gijlers et al. 2009). The study contrasted 3 conditions. In one condition, students received only instruction about RIDE. In a second condition, students received both instruction and the support of a joint reflection tool. And in the final condition, which served as a control condition, students received neither form of support. The collaboration reflection tool prompted joint reflection during collaboration with a focus on self- and peer-assessment as well as goal setting. The interface presented questions for participants to answer and made supporting information for these reflection activities available through button clicks. Differential support was offered depending upon the phase, where the phases included Feed Up, Feed Back, and Feed Forward.

The findings regarding the effect of instruction prior to collaboration are somewhat surprising, though they are consistent with some recent work related to the contrast between implicit and explicit scaffolding for collaboration (Wang et al. 2017b). The Eshuis and colleagues article similarly highlights the unique value of explicit scaffolding through technology of collaborative processes during collaboration, and the synergistic effect of bringing implicit and explicit forms of support together.

\section{Instrumental genesis in the design studio}

In the final two articles, technology resources become a creative medium for students in the midst of collaboration. For example, Lucila Carvalho, Roberto Martinez-Maldonado, and Peter Goodyear illustrate the theory of Instrumental Genesis (IG) as a framework for understanding how collaborators enact design practices in the midst of collaboration. This theoretical work offers a perspective on appreciating the role of technology in collaboration as a first-class contribution. 
Adding to an existing foundation of research in the journal investigating collaboration in connection with interactive surfaces (Tissenbaum et al. 2017), and, building on a foundation in Activity Theory (Nardi 1996; Engeström 1999; Kaptelinin 2005), IG models the joint evolution of artifacts with their uses within specific collaborative environments. An important concept is how artifacts mediate joint activity. The article specifically focuses on four forms of such mediation, namely Epistemic, Pragmatic, Reflexive, and Inter-personal. In this work, Carvalho and colleagues investigate specifically what has been termed the preparation phase in CSCL (Lonchamp 2012), which involves three sets of stakeholders, namely the students, the instructors, and design researchers. Each of these groups of stakeholders use technology support to carry out their role within this landscape.

The work highlights a type of Educational Design Research Studio (EDRS). The specific EDRS featured in this article is equipped with a range of technologies including interactive surfaces, writable walls, an interactive whiteboard, a data projector, and iPads. These design studios are meant to support collaborative efforts of interdisciplinary design teams comprising three to eight people working over a workday or less. The paper highlights the intention behind the design of the space, even down to the positioning of teach tool, in terms of the intended function of the tool for supporting collaborative design practices.

Two in depth qualitative studies are presented in the article, which offer up-close insights into design processes as well as the role of design patterns in the work. Each study features multiple teams engaged in course redesign activities. The practical goal of this work is to provide insights to educational designers to improve their work.

\section{Imagining with improvised representations in cscl environments}

A study of scientific reasoning has been a mainstay in the field of learning sciences (Matuk and Linn 2018). In particular, the differing affordances for support of scientific reasoning has been a running theme in the journal throughout its history, and including in the past year (Ingulfsen et al. 2018). In the final article, technology resources are used as a creative medium out of which representations with communicative affordances are constructed in the midst of collaboration. Rolf Steier, Magdalena Kersting, and Kenneth Silseth investigate practices within groups to creatively fashion multi-modal representations to aid in challenging collaborative reasoning tasks.

The technology highlight in this article is the extent to which technologies associated with different modalities are associated with different affordances that can be leveraged and combined within collaboration. One creative aspect of the thinking behind the article is its leveraging of Lakoff and Johnson's Conceptual Metaphor Theory (Lakoff and Johnson 2008) to explain how embodiment influences student communicative practices in collaboration. This perspective, grounded in ideas related to embodiment, explains how appropriation of representations takes on an organic character in which there is some regularity and systematicity, and yet it is highly creative, which introduces a measure of irregularity and arbitrariness. Just like verbal metaphors, multi-modal representations serve as metaphors that create an intersubjective space to house joint reasoning. They create affordances within interaction that guide and shape the direction the interaction takes. Another creative aspect of the work is its emphasis on student generated representations, which has been a less common focus in the field, though there has been some prior work in this area (Prain and Tytler 2012) and the related area of invented representations (Enyedy 2005). 
The context of the study reported in this paper is a web-based learning module housing a map task featuring two- and three-dimensional spatial representations. The task was chosen specifically because it had been observed in prior work to encourage student creation of new representations in the midst of collaboration. Sociocultural theory is used as a lens for understanding the communicative processes the students engaged in using their invented representations. An important contribution of the article is new insights related to joint meaning making that leverage multi-modal resources.

\section{Conclusion}

Tools of many kinds enable the work we do as learning scientists, and might even be argued to be of particular importance in the field of CSCL. The recent Wise and Schwarz (2017) article characterizing the field and presenting seven provocations listed an effort to provide a comprehensive set of tools to the field as the first of these. Commentary on this point was contributed in a squib published in the following year (Rummel 2018). On the other hand, the central scientific aim of the CSCL community is to produce theory about human cognition and social processes, and development of methods, tools, and technologies are valued but positioned in a less privileged position among contributions to the field. The four articles of this issue highlight some of the diversity in tool efforts within the field while also illustrating the value of technology contributions as first class citizens within the literature of the field.

\section{References}

Buder, J., \& Bodemer, D. (2008). Supporting controversial CSCL discussions with augmented group awareness tools. International Journal of Computer-Supported Collaborative Learning, 3(2), 123-139.

Engeström, Y. (1999). Activity theory and individual and social transformation. In Engeström, Y., Meittinen, R. \& Punamaki, R. (eds). Perspectives on Activity Theory, Cambridge University Press, pp 19-38.

Enyedy, N. (2005). Inventing mapping: Creating cultural forms to solve collective problems. Cognition and Instruction, 23(4), 427-466.

Gijlers, H., Saab, N., Van Joolingen, W. R., De Jong, T., \& Van Hout-Wolters, B. H. (2009). Interaction between tool and talk: How instruction and tools support consensus building in collaborative inquiry-learning environments. Journal of Computer Assisted Learning, 25(3), 252-267.

Hadwin, A. F., Bakhtiar, A., \& Miller, M. (2018). Challenges in online collaboration: Effects of scripting shared task perceptions. International Journal of Computer-Supported Collaborative Learning, 13, 301. https://doi. org/10.1007/s11412-018-9279-9.

Hattie, J. A., \& Donoghue, G. M. (2016). Learning strategies: A synthesis and conceptual model. NPJ Science of Learning, 1, 16013.

Ingulfsen, L., Furberg, A., \& Strømme, T. A. (2018). Students' engagement with real-time graphs in CSCL settings: Scrutinizing the role of teacher support. International Journal of Computer-Supported Collaborative Learning, 13, 365. https://doi.org/10.1007/s11412-018-9290-1.

Jeong, H., \& Hmelo-Silver, C. E. (2016). Seven affordances of computer-supported collaborative learning: How to support collaborative learning? How can technologies help? Educational Psychologist, 51(2), 247-265.

Kaptelinin, V. (2005). The object of activity: Making sense of the sense-maker. Mind, Culture, and Activity, $12(1), 4-18$.

Lakoff, G., \& Johnson, M. (2008). Metaphors we live by. Chicago: University of Chicago press.

Lonchamp, J. (2012). An instrumental perspective on CSCL systems. International Journal of ComputerSupported Collaborative Learning, 7(2), 211-237.

Matuk, C., \& Linn, M. C. (2018). Why and how do middle school students exchange ideas during science inquiry? International Journal of Computer-Supported Collaborative Learning, 13, 263. https://doi. org/10.1007/s11412-018-9282-1. 
Nardi, B. A. (1996). Studying context: A comparison of activity theory, situated action models, and distributed cognition. In Nardi, B. (ed). Context and consciousness: Activity theory and human-computer interaction, MIT Press. pp 7-16.

Näykki, P., Isohätälä, J., Järvelä, S., Pöysä-Tarhonen, J., \& Häkkinen, P. (2017). Facilitating socio-cognitive and socio-emotional monitoring in collaborative learning with a regulation macro script-an exploratory study. International Journal of Computer-Supported Collaborative Learning, 12(3), 251-279.

Phielix, C., Prins, F. J., \& Kirschner, P. A. (2010). Awareness of group performance in a CSCL-environment: Effects of peer feedback and reflection. Computers in Human Behavior, 26(2), 151-161.

Phielix, C., Prins, F. J., Kirschner, P. A., Erkens, G., \& Jaspers, J. (2011). Group awareness of social and cognitive performance in a CSCL environment: Effects of a peer feedback and reflection tool. Computers in Human Behavior, 27(3), 1087-1102.

Prain, V., \& Tytler, R. (2012). Learning through constructing representations in science: A framework of representational construction affordances. International Journal of Science Education, 34(17), 2751-2773.

Rummel, N. (2018). One framework to rule them all? Carrying forward the conversation started by Wise and Schwarz. International Journal of Computer-Supported Collaborative Learning, 13, 123. https://doi. org/10.1007/s11412-018-9273-2.

Saab, N., van Joolingen, W. R., \& van Hout-Wolters, B. H. (2005). Communication in collaborative discovery learning. British Journal of Educational Psychology, 75(4), 603-621.

Tissenbaum, M., Berland, M., \& Lyons, L. (2017). DCLM framework: understanding collaboration in openended tabletop learning environments. International Journal of Computer-Supported Collaborative Learning, 12(1), 35-64.

Wang, X., Kollar, I., \& Stegmann, K. (2017a). Adaptable scripting to foster regulation processes and skills in computer-supported collaborative learning. International Journal of Computer-Supported Collaborative Learning, 12(2), 153-172.

Wang, X., Wen, M., Rosé, C. P. (2017b). Contrasting explicit and implicit support for transactive exchange in team oriented project based learning, Proceedings of Computer-Supported Collaborative Learning, Philadelphia, PA: International Society of the Learning Sciences.

Wise, A. F., \& Schwarz, B. B. (2017). Visions of CSCL: Eight provocations for the future of the field. International Journal of Computer-Supported Collaborative Learning, 12(4), 423-467.

Publisher's note Springer Nature remains neutral with regard to jurisdictional claims in published maps and institutional affiliations. 